\title{
Periodic Graphs
}

\author{
Chris Godsil \\ Combinatorics \& Optimization \\ University of Waterloo \\ cgodsil@uwaterloo.ca
}

Submitted: Nov 4, 2010; Accepted: Jan 17, 2011; Published: Jan 26, 2011

Mathematics Subject Classification: 05C50, 81P68

\begin{abstract}
Let $X$ be a graph on $n$ vertices with adjacency matrix $A$ and let $H(t)$ denote the matrix-valued function $\exp (i A t)$. If $u$ and $v$ are distinct vertices in $X$, we say perfect state transfer from $u$ to $v$ occurs if there is a time $\tau$ such that $\left|H(\tau)_{u, v}\right|=1$. If $u \in V(X)$ and there is a time $\sigma$ such that $\left|H(\sigma)_{u, u}\right|=1$, we say $X$ is periodic at $u$ with period $\sigma$. It is not difficult to show that if the ratio of distinct nonzero eigenvalues of $X$ is always rational, then $X$ is periodic. We show that the converse holds, from which it follows that a regular graph is periodic if and only if its eigenvalues are distinct. For a class of graphs $X$ including all vertex-transitive graphs we prove that, if perfect state transfer occurs at time $\tau$, then $H(\tau)$ is a scalar multiple of a permutation matrix of order two with no fixed points. Using certain Hadamard matrices, we construct a new infinite family of graphs on which perfect state transfer occurs.
\end{abstract}

\section{Introduction}

Let $X$ be a graph with adjacency matrix $A$. We define the matrix function $H(t)$ by

$$
H(t):=\exp (i t A):=\sum_{n \geq 0} i^{n} A^{n} \frac{t^{n}}{n !} .
$$

We note that $t$ is a real variable

$$
H(t)^{*}=\exp (-i A t)=H(t)^{-1}
$$

and therefore $H(t)$ is a unitary matrix. We have $H(0)=I$ and

$$
H(s+t)=H(s) H(t) .
$$


We say that graph is periodic with respect to the vector $z$ if there is a real number $\tau$ such that $H(\tau) z$ is a scalar multiple of $z$. (Since $H(t)$ is unitary, this scalar will have absolute value 1.) We say that $X$ is periodic relative to the vertex $u$ if it is periodic relative to the standard basis vector $e_{u}$ or, equivalently if there is a time $\tau$ such that $\left|H(\tau)_{u, u}\right|=1$. We say that $X$ itself is periodic if there is a time $\tau$ such that $H(\tau)$ is diagonal.

If there are basis vectors $z_{1}, \ldots, z_{n}$ such that $X$ is periodic with period $\tau_{r}$ relative to the vector $z_{r}$ for $r=1, \ldots, n$, then it follows that $X$ is periodic, with period dividing the product of the $\tau_{i}$ 's.

We say we have perfect state transfer if there are distinct vertices $u$ and $v$ in $X$ and a time $\tau$ such that

$$
\left|H(\tau)_{u, v}\right|=1 .
$$

(If this holds then $H(\tau)_{u, v}$ is the only non-zero entry in its row and column.) Christandl, Datta, Dorlas, Ekert, Kay and Landahl [5] prove that we have perfect state transfer between the end-vertices of paths of length one and two, and between vertices at maximal distance in Cartesian powers of these graphs. A graph exhibiting perfect state transfer between two vertices $u$ and $v$ models a network of quantum particles with fixed couplings, in which the state of the particle in $u$ can be transferred to the particle in $v$ without any information loss.

This paper is an attempt to extend some of the results in the above paper, and in the more recent work by Saxena, Severini and Shparlinski [13]. We prove that a regular graph is periodic if and only if its eigenvalues are integers. We also show for a wide class of regular graphs (including all vertex-transitive graphs and all distance-regular graphs) that if perfect state transfer occurs, then there is a time $\tau$ such that $H(\tau)$ is a scalar multiple of a permutation matrix of order two with zero diagonal. (And hence the number of vertices in the graph must be even.) Finally we present a new infinite class of antipodal distance-regular graphs where perfect state transfer occurs.

\section{Eigenvalues}

We are going to derive consequences for the eigenvalues of $A$ when $X$ is periodic, but first we show that periodicity and perfect state transfer are related.

2.1 Lemma. If perfect state transfer from $u$ to $v$ occurs at time $\tau$, then $X$ is periodic at $u$ and $v$ with period $2 \tau$.

Proof. Assume $H=H(\tau)$ and suppose that

$$
H(\tau) e_{u}=\gamma e_{v}
$$

where $\|\gamma\|=1$. Then $H_{v, u}=\gamma$ (because $H(t)$ is symmetric) and therefore $H e_{v}=\gamma e_{u}$. Now

$$
\gamma^{2} e_{u}=\gamma H e_{v}=H^{2} e_{u}
$$

and similarly $H^{2} e_{v}=e_{v}$. 
Our main tool in this paper is the spectral decomposition of a symmetric matrix. Suppose $A$ is a symmetric matrix with eigenvalues $\theta_{1}, \ldots, \theta_{d}$, and let $E_{r}$ be the matrix that represents orthogonal projection on the eigenspace associated with $\theta_{r}$. If $f$ a complexvalued function defined on the eigenvalues of $A$, we have

$$
f(A)=\sum_{r=1}^{d} f\left(\theta_{r}\right) E_{r} .
$$

In particular,

$$
H(t)=\sum_{r} \exp \left(i t \theta_{r}\right) E_{r}
$$

Note that the scalars $f\left(\theta_{r}\right)$ are the eigenvalues of $f(A)$ and that $\sum E_{r}=I$. We will also make use of the fact that $f(A)$ is necessarily a polynomial in $A$ (because each projection $E_{r}$ is).

Assume now that $A$ is the adjacency matrix of a graph $X$ on $v$ vertices. If $x$ is a vector in $\mathbb{R}^{v}$, we say that an eigenvalue $\theta_{r}$ of $X$ is in the eigenvalue support of $x$ if $E_{r} x \neq 0$. The case of interest to us will be when $x$ is the characteristic vector of a subset $S$ of $V(X)$. The eigenvalue support of a non-zero vector cannot be empty and, if $x \neq 0$, we define the dual degree of $x$ to be one less than the size of its eigenvalue support.

2.2 Theorem. Let $X$ be a graph and let $u$ be a vertex in $X$ at which $X$ is periodic. If $\theta_{k}, \theta_{\ell}, \theta_{r}, \theta_{s}$ are eigenvalues in the support of $e_{u}$ and $\theta_{r} \neq \theta_{s}$,

$$
\frac{\theta_{k}-\theta_{\ell}}{\theta_{r}-\theta_{s}} \in \mathbb{Q} .
$$

Proof. Suppose the period of $X$ at 1 is $\tau$. Then

$$
H(\tau)_{1,1}=\gamma
$$

where $|\gamma|=1$. Since $H(\tau)$ is unitary, this implies that we have

$$
H(\tau)=\left(\begin{array}{cc}
\gamma & 0 \\
0 & H_{1}
\end{array}\right)
$$

where $H_{1}$ is unitary. Hence

$$
\gamma e_{1}=H(\tau) e_{1}=\sum_{r} \exp \left(i \tau \theta_{r}\right) E_{r} e_{1}
$$

The non-zero vectors $E_{r} e_{1}$ are pairwise orthogonal and so linearly independent, whence we see that

$$
\exp \left(i \tau \theta_{r}\right)=\gamma
$$

for each eigenvalue $\theta_{r}$ in the support of $e_{1}$.

Consequently of $\theta_{r}$ and $\theta_{s}$ are in the eigenvalue support of $e_{1}$, then

$$
\exp \left(i \tau\left(\theta_{r}-\theta_{s}\right)\right)=1
$$

and so $\tau\left(\theta_{r}-\theta_{s}\right)$ is an integral multiple of $2 \pi$. This proves the theorem. 
2.3 Corollary. If $X$ is periodic at $u$ and there are two integer eigenvalues in the support of $e_{u}$, then all eigenvalues in its support are integers.

Proof. Suppose $k$ and $\ell$ are distinct integer eigenvalues in the support of $e_{u}$. By the theorem

$$
\frac{\theta_{r}-\ell}{k-\ell} \in \mathbb{Q}
$$

and therefore $\theta_{r}$ is rational. Since $\theta_{r}$ is an algebraic integer, it must be an integer.

The condition in the previous theorem is known as the ratio condition. Christandl et al [5] stated that if perfect state transfer takes place on a path, then its eigenvalues must satisfy the ratio condition. Similarly Saxena et al [13] proved that if a circulant graph is periodic, its eigenvalues must satisfy the ratio condition.

We can derive these results from ours, taking the second first. A circulant graph is vertex transitive and so if $E_{r} e_{u}=0$ for some vertex $u$, then for all vertices $u$, we have $E_{r} e_{u}=0$. But this implies that $E_{r}=0$, and so we conclude that the support of $e_{u}$ is the set of all eigenvalues of $X$. Hence the eigenvalues of a periodic circulant satisfy the ratio condition.

Now paths. From the spectral decomposition we have

$$
(t I-A)_{u, u}^{-1}=\sum_{r}\left(t-\theta_{r}\right)^{-1}\left(E_{r}\right)_{u, u}
$$

while standard matrix theory yields that

$$
(t I-A)_{u, u}^{-1}=\frac{\phi(X \backslash u, t)}{\phi(X, t)}
$$

Since

$$
\left(E_{r}\right)_{u, u}=e_{u}^{T} E_{r} e_{u}=e_{u}^{T} E_{r}^{2} e_{u}=e_{u}^{T} E_{r}^{T} E_{r} e_{u},
$$

we see that $\left(E_{r}\right)_{u, u}$ is not zero if and only if $E_{r} e_{u}$ is not zero, that is, if and only if $\theta_{r}$ is in the support of $e_{u}$. Therefore the dual degree of $e_{u}$ is equal to the number of poles of the rational function $\phi(X \backslash u, t) / \phi(X, t)$, less 1 . The characteristic polynomial of the path $P_{n}$ on $n$ vertices satisfies the recurrence

$$
\phi\left(P_{n+1}, t\right)=\phi\left(P_{n}, t\right)-\phi\left(P_{n-1}, t\right), \quad(n \geq 1) ;
$$

since $\phi\left(P_{0}, t\right)=1$ and $\phi\left(P_{1}, t\right)=t$, it follows that $\phi\left(P_{n}, t\right)$ and $\phi\left(P_{n-1}, t\right)$ are coprime. So our rational function has $n$ distinct zeros, and again we find that the eigenvalue support of a vertex of degree one in a path is the set of all eigenvalues of the path.

\section{Periodicity}

It follows from the spectral decomposition

$$
H(\tau)=\sum_{r} \exp \left(i \tau \theta_{r}\right) E_{r}
$$


that if the eigenvalues of $X$ are integers, then

$$
H(2 \pi)=\sum_{r} E_{r}=I
$$

and therefore $X$ is periodic with period $2 \pi$. This shows that the path $P_{2}$ is periodic. However, as Christandl et al noted, $P_{3}$ is periodic and its eigenvalues are

$$
-\sqrt{2}, 0, \sqrt{2} \text {. }
$$

Thus integrality is sufficient, but not necessary. There is a more general condition which is necessary and sufficient.

3.1 Theorem. Suppose that the eigenvalues of $X$ are $\theta_{1}, \ldots, \theta_{d}$. Then $X$ is periodic if and only if the ratio of any two non-zero eigenvalues is rational.

Proof. We first show that the given condition is sufficient. If it holds and $\theta_{s} \neq 0$, there are rational numbers $q_{1}, \ldots, q_{r}$ such that $\theta_{r}=q_{r} \theta_{s}$ and therefore there is an integer $N$ and integers $k_{1}, \ldots, k_{r}$ such that $N \theta_{r}=k_{r} \theta_{s}$. Hence if we take

$$
\tau=\frac{2 N \pi}{\theta_{s}}
$$

we have

$$
\exp \left(i \tau \theta_{r}\right)=\exp \left(2 i k_{r} \pi\right)=1
$$

for all $r$, and consequently $X$ is periodic.

Conversely, suppose $X$ is periodic with period $\tau$. Then $H(\tau)$ is diagonal and commutes with $A$. If we view the diagonal of $H(\tau)$ as a function on $V(X)$, it must be constant on the connected components of $X$.

If $X$ is connected it follows that there is a complex number $\gamma$ such that $|\gamma|=1$ and $H(\tau)=\gamma I$. Since the sum of the eigenvalues of $X$ is zero, the product of the eigenvalues of $H(\tau)$ is one, and thus if $v=|V(X)|$, then

$$
1=\operatorname{det}(H(\tau))=\gamma^{v}
$$

Therefore $H(v \tau)=I$, and so all eigenvalues of $H(v \tau)$ are equal to one. If $X$ is not connected, we can apply this argument to deduce that each diagonal entry of $H(\tau)$ is an $m$-th root of unity, for some integer $m$, and therefore if $w$ is the least common multiple of the sizes of the connected components of $X$, then $H(w \tau)=I$.

Hence

$$
\exp \left(i w \tau \theta_{r}\right)=1
$$

for all $r$, and there are integers $\ell_{1}, \ldots, \ell_{r}$ such that

$$
w \tau \theta_{r}=2 \ell_{r} \pi
$$

This implies that, for all $r$ and $s$,

$$
\frac{\theta_{r}}{\theta_{s}}=\frac{\ell_{r}}{\ell_{s}} \in \mathbb{Q}
$$

THE EleCtronic journal of COMBInAtorics 18 (2011), \#P23 
3.2 Lemma. If the ratios of the non-zero eigenvalues of $X$ are rational, then the square of an eigenvalue is an integer.

Proof. If the condition of the lemma holds and $\theta_{s} \neq 0$, there are rationals $q_{1}, \ldots, q_{r}$ such that $\theta_{r}=q_{r} \theta_{s}$. Since the sum of the squares of the eigenvalues of $A$ is equal to twice the number of edges, we see that $\theta_{s}^{2}$ is rational. Since $\theta_{s}$ is an algebraic integer, $\theta_{s}^{2}$ is thus a rational algebraic integer, and accordingly it is actually an integer.

3.3 Corollary. A graph is $X$ is periodic if and only if either:

(a) The eigenvalues of $X$ are integers, or

(b) The eigenvalues of $X$ are rational multiples of $\sqrt{\Delta}$, for some square-free integer $\Delta$.

If the second alternative holds, $X$ is bipartite.

Proof. The stated conditions are sufficient, and so we show they are necessary.

Suppose $k$ is a non-zero integer eigenvalue of $X$. Then $\theta_{r} / k \in \mathbb{Q}$ and so $\theta_{r} \in \mathbb{Q}$. Since $\theta_{r}$ is an algebraic integer, it is therefore an integer. If no non-zero eigenvalue of $X$ is an integer, then by the theorem each eigenvalue is a rational multiple of the spectral radius $\theta_{1}$, say $\theta_{i}=m_{i} \theta_{1}$.

Also by the theorem $\theta_{1}^{2} \in \mathbb{Z}$, whence it follows that $t^{2}-\theta_{1}^{2}$ is the minimal polynomial of $\theta_{1}$ over the rationals, and therefore $-\theta_{1}$ is an eigenvalue of $X$. By the Perron-Frobenius theory, this implies that $X$ is bipartite.

\section{Coherent Algebras}

A coherent algebra is a real or complex algebra of matrices that contains the all-ones matrix $J$ and is closed under Schur multiplication, transpose and complex conjugation. (The Schur product $A \circ B$ of two matrices $A$ and $B$ with same order is defined by

$$
(A \circ B)_{i, j}=A_{i, j} B_{i, j} .
$$

It has been referred to as the "bad student's product".) A coherent algebra always has a basis of 01-matrices and this is unique, given that its elements are 01-matrices. This set of matrices determines a set of directed graphs and the combinatorial structure they form is known as a coherent configuration. When we say that a graph $X$ is a "graph in a coherent algebra", we mean that $A(X)$ is a sum of distinct elements of the 01-basis.

A coherent algebra is homogeneous if the identity matrix is an element of its 01-basis. If $M$ belongs to a homogeneous coherent algebra, then

$$
M \circ I=\mu I
$$

for some scalar $\mu$. Hence the diagonal of any matrix in the algebra is constant. If $A$ is a 01matrix in the algebra, the diagonal entries of $A A^{T}$ are the row sums of $A$. Therefore all row 
sums and all column sums of any matrix in the 01-basis are the same, and therefore this holds for each matrix in the algebra. In particular we can view the non-identity matrices as adjacency matrices of regular directed graphs. Any directed graph in a homogeneous coherent algebra must be regular.

We consider two classes of examples. First, if $\mathcal{P}$ is a set of permutation matrices of order $n \times n$, then the commutant of $\mathcal{P}$ in $\operatorname{Mat}_{n \times n}(\mathbb{C})$ is Schur-closed. Therefore it is a coherent algebra, and this algebra is homogeneous if and only the permutation group generated by $\mathcal{P}$ is transitive. Thus any graph whose automorphism group acts transitively on its vertices belongs to a coherent algebra

For second class of examples, if $X$ is a graph we define the $r$-th distance graph $X_{r}$ of $X$ to be the graph with $V\left(X_{r}\right)=V(X)$, where vertices $u$ and $v$ are adjacent in $X_{r}$ if and only if they are at distance $r$ in $X$. So if $X$ has diameter $d$, we have distance graphs $X_{1}, \ldots, X_{d}$ with corresponding adjacency matrices $A_{1}, \ldots, A_{d}$. If we use $A_{0}$ to denote $I$, then the graph $X$ is distance regular if the matrices $A_{0}, \ldots, A_{d}$ are the 01-basis of a homogeneous coherent algebra. (It must be admitted that this is not the standard definition.) We will refer to the matrices $A_{i}$ as distance matrices.

A commutative coherent algebra is the same thing as a Bose-Mesner algebra of an association scheme. We will not go into this here, but we do note that the coherent algebra belonging to a distance-regular graph is commutative.

4.1 Theorem. If $X$ is a graph in a coherent algebra with vertices $u$ and $v$, and perfect state transfer from $u$ to $v$ occurs at time $\tau$, then $H(\tau)$ is a scalar multiple of a permutation matrix with order two and no fixed points that lies in the centre of the automorphism group of $X$.

Proof. First, if $A=A(X)$ where $X$ is a graph in a homogeneous coherent algebra then, because it is a polynomial in $A$, the matrix $H(t)$ lines in the algebra for all $t$. Hence if

$$
\left|H(\tau)_{u, v}\right|=1
$$

it follows that $H(\tau)=\xi P$ for some complex number $\xi$ such that $|\xi|=1$ and some permutation matrix $P$. Since $A$ is symmetric, so is $H(t)$ for any $t$, and therefore $P$ is symmetric. So

$$
P^{2}=P P^{T}=I
$$

and $P$ has order two. Since $P$ has constant diagonal, its diagonal is zero and it has no fixed points. As $P$ is a polynomial in $A$, it commutes with any automorphism of $X$ and hence is central.

4.2 Corollary. If $X$ is a graph in a coherent algebra with vertices $u$ and $v$ and perfect state transfer from $u$ to $v$ occurs at some time, then the number of vertices of $X$ is even.

Proof. Since $P^{2}=I$ and the diagonal of $P$ is zero, the number of columns of $P$ is even. 
Saxena et al [13] proved this corollary for circulant graphs. A homogeneous coherent algebra is imprimitive if there is a non-identity matrix in its 01-basis whose graph is no connected, otherwise it is primitive. If the algebra is the commutant of a transitive permutation group, it is imprimitive if and only the group is imprimitive as a permutation group. The above corollary implies that if perfect state transfer takes place on a graph from a homogeneous coherent algebra, the algebra is imprimitive.

Note that our corollary holds for any vertex-transitive graph, and for any distanceregular graph.

\section{$5 \quad$ Walk-Regular Graphs}

A graph is walk regular if its vertex-deleted subgraphs $X \backslash u$ are all cospectral. We have the following result which implies that any graph in a coherent configuration is walk regular. It follows immediately from Theorem 4.1 in [7].

5.1 Lemma. A graph $X$ with adjacency matrix $A$ is walk regular if and only if the diagonal entries of $A^{k}$ are constant for all non-negative integers $k$.

Any connected regular graph with at most four distinct eigenvalues is walk regular (see Van Dam [15]). Note that a walk-regular graph is necessarily regular. The graph in Figure 1 is walk regular but not vertex transitive. This graph does not lie in a homogeneous coherent algebra - the row sums of the Schur product

$$
A \circ\left(A^{2}-4 I\right) \circ\left(A^{2}-4 I-J\right)
$$

are not all the same.

Suppose perfect state transfer takes place from $u$ to $v$ at time $\tau$. Then there is a complex number $\gamma$ where $|\gamma|=1$ such that

$$
H(2 \tau)_{u, v}=H(2 \tau)_{v, u}=\gamma
$$

As $|\gamma|=1$, we see that $H_{u, u}=0$ and, as $X$ is walk-regular, all diagonal entries of $H$ are zero and in particular, $\operatorname{tr}(H(\tau))=0$. Since

$$
H(2 \tau)_{u, u}=\gamma^{2}
$$

we also see that that $H(2 \tau)=\gamma^{2} I$. We conclude that the eigenvalues of $H(\tau)$ are all $\pm \gamma$ and, since $\operatorname{tr}(H(\tau))=0$, both $\gamma$ and $-\gamma$ have multiplicity $|V(X)| / 2$. (Accordingly $|V(H)|$ must be even.)

If $m_{\theta}$ denotes the multiplicity of the eigenvalue $\theta$, then

$$
\operatorname{tr}(H(t))=\sum_{\theta} m_{\theta} \exp (i t \theta)
$$

and thus we may define the Laurent polynomial $\mu(z)$ by

$$
\mu(z):=\sum_{\theta} m_{\theta} z^{\theta} .
$$




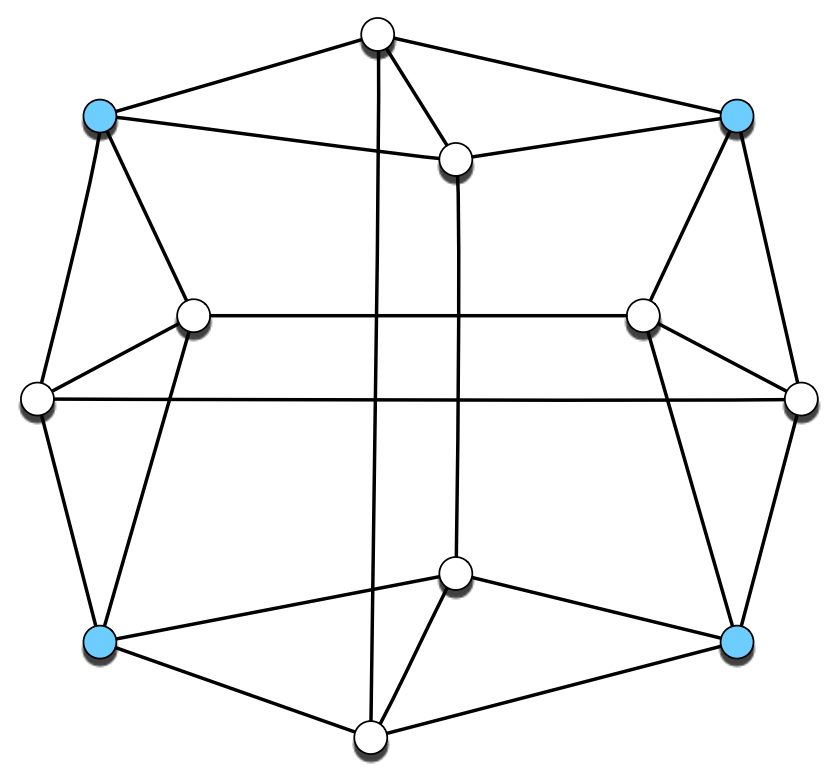

Figure 1: A Walk-Regular Graph

Here $z=\exp (i t)$ and we have made use of the fact that $X$ is periodic and hence its eigenvalues are integers. We call $\mu(z)$ the multiplicity enumerator of $X$.

5.2 Lemma. Let $X$ be a walk-regular graph with integer eigenvalues. If perfect state transfer occurs on $X$, then $\mu(z)$ has an eigenvalue on the unit circle of the complex plane.

Proof. The trace of $H(t)$ is zero if and only if $\mu(\exp (i t))=0$.

The characteristic polynomial of the graph in Figure 1 is

$$
(t-4)(t-2)^{3} t^{3}(t+2)^{5}
$$

and its multiplicity enumerator is

$$
\mu(z)=z^{-2}\left(z^{6}+3 z^{4}+3 z^{2}+5\right) .
$$

This polynomial has no roots on the unit circle, and we conclude that perfect state transfer does not occur.

We see no reason to believe that, if perfect state transfer occurs on a walk-regular graph at time $\tau$, then $H(\tau)$ must be a multiple of a permutation matrix. (But we do not have an example.)

For more information on walk-regular graphs, see [7]. The computations in this section were carried out in sage [14]. Van Dam [15] studies regular graphs with four eigenvalues, as we noted these provide examples of walk-regular graphs. 


\section{Hypercubes}

The $n$-cube was one of the first cases where perfect state transfer was shown to occur (see [5]). The $n$-cube is an example of a distance-regular graph, and in this section we establish some of the consequences of our work for distance-regular graphs.

Suppose $X$ is a distance-regular graph with diameter $d$ and distance matrices

$$
A_{0}, \ldots, A_{d}
$$

It has long been known that if $X$ is imprimitive then either $X_{2}$ is not connected or $X_{d}$ is not connected (see [3, Chapter 22] or [4, Section 4.2]). For the $n$-cube, neither $X_{2}$ nor $X_{n}$ are connected. If $X_{2}$ is not connected then $X$ is bipartite and $X_{m}$ is not connected if $m$ is even. Here we will be concerned with the case where $X_{d}$ is not connected. In this case it follows from the work just cited that the components of $X_{d}$ must be complete graphs of the same size, and that two vertices of $X$ lie in the same component of $X_{d}$ if and only if they are at distance $d$ in $X$. The components are called the antipodal classes of $X$; note these may have size greater than two. If $X_{d}$ is not connected, we say that $X$ is an antipodal distance regular graph.

As already noted the $n$-cubes provide natural examples of distance-regular graphs. The $n$-cube has diameter $n$, and its vertices can be partitioned into pairs such that two vertices in the same pair are at distance $n$, while vertices in different pairs are at distance less than $n$, hence it is antipodal.

Our next lemma is more or less a specialization of Corollary 4.2 to the case of distanceregular graphs.

6.1 Lemma. Suppose $X$ is a distance-regular graph with diameter $d$. If perfect state transfer from $u$ to $v$ occurs at time $\tau$, then $X$ is antipodal with antipodal classes of size two and $H(\tau)=X_{d}$.

We outline a proof that perfect state transfer occurs on the $n$-cube. In the following section we will use much the same argument to provide a second infinite class of examples.

The $n$-cube gives rise to an association scheme, that is, a commutative coherent configuration as follows. Let $X$ be the graph of the $n$-cube, and view its vertices as binary vectors of length $n$. Let $X_{i}$ be the graph with the same vertex set as $X$, where two vertices are adjacent if and only if they differ in exactly $i$ positions. Thus $X_{1}=X$ and two vertices are adjacent in $X_{i}$ if and only they are at distance $i$ in $X$. Let $A_{i}$ be the adjacency matrix of $X_{i}$ and set $A_{0}=I$. Then $A_{0}, \ldots, A_{n}$ are symmetric 01-matrices and

$$
\sum_{r=0}^{n} A_{r}=J .
$$

It is known that these matrices commute and that their span is closed under matrix multiplication. Hence they form a basis for a commutative coherent algebra of dimension $d+1$. Since this algebra is commutative and semisimple, it has a second basis

$$
E_{0}, \ldots, E_{n}
$$


of matrix idempotents. These are real symmetric matrices such that $E_{i} E_{j}=\delta_{i, j}$ and

$$
\sum_{i=0}^{n} E_{i}=I
$$

These idempotents represent orthogonal projection onto the eigenspaces of the algebra and hence there are scalars $p_{i}(j)$ such that $A_{i} E_{j}=p_{i}(j) E_{j}$. Consequently

$$
A_{i}=A_{i} I=A_{i} \sum_{r=0}^{n} E_{r}=\sum_{r=0}^{n} p_{i}(r) E_{r}
$$

The scalars $p_{i}(j)$ are known as the eigenvalues of the scheme.

On the other hand, the matrices $A_{0}, \ldots, A_{n}$ are a basis for the algebra and thus there are scalars $q_{i}(j)$ such that

$$
E_{j}=2^{-n} \sum_{r=0}^{n} q_{j}(r) A_{r}
$$

The scalars $q_{i}(j)$ are the dual eigenvalues of the scheme.

If $f$ is any function defined on the eigenvalues of the scheme then

$$
f\left(A_{1}\right)=\sum_{r=0}^{n} f\left(p_{1}(r)\right) E_{r}
$$

For the $n$-cube, $p_{1}(r)=n-2 r$ and accordingly

$$
H(t)=\sum_{r=0}^{n} \exp (i(n-2 r) t) E_{r}=\exp (\text { int }) \sum_{r=0}^{n} \exp (-2 i r t) E_{r} .
$$

If we set $t=\pi$, we then have

$$
H(\pi)=(-1)^{n} \sum_{r=0}^{n} E_{r}=(-1)^{n} I .
$$

Thus the $n$-cube is periodic with period $\pi$. Now try $n=\pi / 2$. Then

$$
H(\pi / 2)=i^{n} \sum_{r=0}^{n}(-1)^{r} E_{r}
$$

From, for example, [12, Section 21.3] we know that

$$
\sum_{r=0}^{n}(-1)^{r} E_{r}=A_{d}
$$

and we conclude that perfect state transfer occurs at time $\pi / 2$.

In [11], Jafarizadeh and Sufiani give examples of perfect state transfer on distanceregular graphs that arise when a weighted adjacency matrix is used in place of the ordinary adjacency matrix. 


\section{Hadamard Matrices}

We now show how to use a special class of Hadamard matrices to construct distanceregular graphs of diameter three with perfect state transfer.

The Hadamard matrices $H$ we want must be as follows:

(a) Symmetric: $H=H^{T}$.

(b) Regular: all row and column sums are equal.

(c) Constant diagonal: all entries on the diagonal are equal.

Suppose $H$ is $n \times n$ and satisfies this constellation of conditions. If its rows sum to $c$, then $H J=c J$ and $H^{2} J=c^{2} J$. Since

$$
H^{2} I=H H^{T}=n I
$$

whence $c= \pm \sqrt{n}$. It follows that there are two possibilities: either the diagonal entries and the rows sums of $H$ have the same sign, or they do not. To handle this we assign a parameter $\epsilon=\epsilon(H)$ to $H$, by defining $\epsilon$ to be the sign of the product of the row sum of $H$ with a diagonal entry. Whatever the value of $\epsilon(H)$, the order of $H$ must be a square. For a recent useful paper on these Hadamard matrices, see Haemers [10].

We remark that if the row sums of a Hadamard matrix are all equal then its columns sums must all be equal as well, and again the order of the matrix must be a square. Similarly, a symmetric Hadamard matrix with constant diagonal must have square order. If $H$ and $K$ are regular symmetric Hadamard matrices with constant diagonal, then so is their Kronecker product $H \otimes K$ and

$$
\epsilon(H \otimes K)=\epsilon(H) \epsilon(H)
$$

Finally if $H$ is a regular Hadamard matrix of order $n \times n$ with constant diagonal, and $P$ is the permutation matrix on $\mathbb{R}^{n} \otimes \mathbb{R}^{n}$ defined by

$$
P(u \otimes v)=v \otimes u
$$

then $P\left(H \otimes H^{T}\right)$ is a symmetric regular Hadamard matrix with constant diagonal.

Suppose $H$ is a regular symmetric $n \times n$ Hadamard matrix with constant diagonal. Replacing $H$ by $-H$ is necessary, we may assume that the diagonal entries of $H$ are equal to 1 , and then the matrix

$$
\frac{1}{2}(J+H)-I
$$

is the adjacency matrix of a regular graph (in fact a strongly regular graph). Unfortunately the graphs that result are not the ones we need. Our aim rather is to construct an antipodal distance-regular graph of diameter three on $2 n$ vertices from $H$. (These graphs are member of a class known as regular two-graphs. An introductory treatment is offered in $[8$, Chapter 11]) 
We construct our graphs as follows. Let $H$ be a symmetric Hadamard matrix of order $n \times n$ with constant row sum and with all diagonal entries equal to 1 . We construct a graph $X(H)$ with vertex set

$$
\{1, \ldots, n\} \times\{0,1\}
$$

where $(i, a)$ is adjacent to $(j, b)$ if and only if $i \neq j$ and

$$
H_{i, j}=(-1)^{a+b}
$$

Clearly this gives us a regular graph $X(H)$ on $2 n$ vertices with valency $n-1$. It is not hard to check that its diameter is three, and two vertices are at distance three if and only if they are of the form

$$
(i, 0),(i, 1)
$$

for some $i$. Suppose $A$ is the adjacency matrix of $X(H)$. The space $S$ of vectors that are constant on the antipodal classes of $X(H)$ has dimension $n$ and the matrix representing the action of $A$ on $S$ is the $n \times n$ matrix $J_{I}$. Its eigenvalues are $n-1$ and -1 with respective multiplicities 1 and $n-1$. The orthogonal complement of $S$ is also $A$-invariant and the matrix representing the action of $A$ is $H$.

If we write $H$ as $I+C-D$ where $C$ and $D$ are 01-matrices, then

$$
A(X(H))=C \otimes\left(\begin{array}{ll}
1 & 0 \\
0 & 1
\end{array}\right)+D \otimes\left(\begin{array}{ll}
0 & 1 \\
1 & 0
\end{array}\right)
$$

and the eigenvalues of $A$ are the eigenvalues of the matrices

$$
C+D=J-I, \quad C-D=H-I .
$$

Therefore the eigenvalues of $A$ are $n-1,-1$ and the eigenvalues of $H-I$. Since $H^{2}=n I$, we see that the eigenvalues of $A$ are

$$
n-1,-1, \sqrt{n}-1,-\sqrt{n}-1 \text {. }
$$

If $a$ is the multiplicity of $\sqrt{n}$ as an eigenvalue of $F$, then

$$
n=\operatorname{tr}(A)=a \sqrt{n}-(n-a) \sqrt{n}
$$

whence

$$
a=\frac{1}{2}(n+\sqrt{n})
$$

it follows that

$$
\begin{aligned}
\mu(z) & =z^{n-1}+(n-1) z^{-1}+\frac{1}{2}(n+\sqrt{n}) z^{\sqrt{n}-1}+\frac{1}{2}(n-\sqrt{n}) z^{-\sqrt{n}-1} \\
& =z^{-\sqrt{n-1}}\left(z^{n+\sqrt{n}}+(n-1) z^{\sqrt{n}}+\frac{1}{2}(n-\sqrt{n}) z^{2 \sqrt{n}}+\frac{1}{2}(n-\sqrt{n})\right) .
\end{aligned}
$$


If $z^{\sqrt{n}}=-1$, then $z^{2 \sqrt{n}}=1$ and, since $\sqrt{n}$ is even,

$$
z^{n-\sqrt{n}}=\left(z^{\sqrt{n}}\right)^{\sqrt{n}} z^{-\sqrt{n}}=-1
$$

Therefore if $z^{\sqrt{n}}=-1$, then $\mu(z)=0$ and if $z=e^{i t}$ then

$$
H(t)=-E_{n-1}-E_{(-1)}+E_{(-1+\sqrt{n})}+E_{(-1-\sqrt{n})} .
$$

Let $F$ be the direct sum of $n$ copies of the matrix

$$
\frac{1}{2}\left(\begin{array}{ll}
1 & 1 \\
1 & 1
\end{array}\right) .
$$

Then $\operatorname{rk}(F)=n$ and $\operatorname{col}(F)$ is the space of functions constant on the antipodal classes of $X(H)$. Since $F^{2}=F$, we find that $E_{(-1)}=F-E_{n-1}$ and consequently

$$
H(t)=I-2 F
$$

So $H(t)$ is a permutation matrix and we have perfect state transfer.

Infinitely many examples of regular symmetric Hadamard matrices with constant diagonal are known, in particular they exist whenever the order $n$ is a power of four. Haemers [10] provides a summary of the state of our knowledge.

\section{Questions and Comments}

The most obvious problem remaining is to characterize the pairs of vertices where perfect state transfer takes place.

Angeles-Canul et al [1] et al give examples of graphs where perfect state transfer occurs but the underlying graph is not periodic; this answered a question we raised in an earlier version of this paper.

Facer, Twamley and Cresser [6] construct certain Cayley graphs for $\mathbb{Z}_{2}^{d}$ on which perfect state transfer; the resulting family of graphs include the $n$-cubes. In [2], it is shown that if $C$ is the connection set for a Cayley graph for $\mathbb{Z}_{2}^{d}$ and $\sigma$ is the sum of the elements of $C$ and $\sigma \neq 0$, then we have perfect state transfer from $v$ to $v+\sigma$ for each element $v$ of $\mathbb{Z}_{2}^{d}$. The $d$-cube has $2^{d}$ edges. It would be interesting to have a good estimate for the minimum number of edges in a graph where perfect state transfer between two vertices at distance $d$ occurs.

I would like to acknowledge a number of very useful discussions with Simone Severini on the topics of this paper. 


\section{References}

[1] Ricardo Javier Angeles-Canul, Rachael M. Norton, Michael C. Opperman, Christopher C. Paribello, Matthew C. Russell and Christino Tamon. Perfect state transfer, integral circulants and join of graphs. Quantum Information and Computation, 10(3\&4):325-342, 2010. Available from http://arxiv.org/abs/0907.2148.

[2] Anna Bernasconi, Chris Godsil, Simone Severini. Quantum Networks on Cubelike Graphs Phys. Rev. A, 78, 052320 (2008). Available from http://arxiv.org/abs/ $0808.0510 \mathrm{v} 1$.

[3] Norman Biggs. Algebraic Graph Theory. Cambridge University Press, Cambridge, second edition, 1993.

[4] A. E. Brouwer, A. M. Cohen, and A. Neumaier. Distance-Regular Graphs. SpringerVerlag, Berlin, 1989.

[5] Matthias Christandl, Nilanjana Datta, Tony C. Dorlas, Artur Ekert, Alastair Kay and Andrew J. Landahl. Perfect transfer of arbitrary states in quantum spin networks. Physical Review A, 71:032312, 2005. Available from: http://arxiv.org/ abs/quant-ph/0411020.

[6] C. Facer, J. Twamley and J. D. Cresser. Quantum Cayley networks of the hypercube. Phys. Rev. A, 77:012334, 2008. Available from: http://arxiv.org/abs/0706.3821.

[7] C. D. Godsil and B. D. McKay. Feasibility conditions for the existence of walk-regular graphs. Linear Algebra Appl., 30:51-61, 1980.

[8] Chris Godsil and Gordon Royle. Algebraic Graph Theory. Springer-Verlag, New York, 2001.

[9] Chris Godsil and Simone Severini. Control by quantum dynamics on graphs. Physical Review A, 81:052316 (2010) Available from http://arxiv.org/abs/0910.5397

[10] W. H. Haemers. Strongly regular graphs with maximal energy. Linear Algebra Appl., 429:2719-2723, 2008.

[11] M. A. Jafarizadeh and R. Sufiani. Perfect state transfer over distance-regular spin networks, 2007. Available from: http://arxiv.org/abs/0709.0755.

[12] F. J. MacWilliams and N. J. A. Sloane. The Theory of Error-Correcting Codes. North-Holland Publishing Co., Amsterdam, 1977.

[13] Nitin Saxena, Simone Severini, and Igor Shparlinski. Parameters of integral circulant graphs and periodic quantum dynamics. Int. J. Quant. Inf., 5:417-430, 2007. Available from: http://arxiv.org/abs/quant-ph/0703236.

[14] William Stein. Sage: Open Source Mathematical Software (Version 3.0). The Sage Group, 2008. http://www . sagemath .org.

[15] Edwin R. van Dam. Regular graphs with four eigenvalues. Linear Algebra Appl., 226/228:139-162, 1995. 